\title{
A CASE OF RIEDEL'S THYROIDITIS
}

\author{
Z. Rajkovaca1, R. Gajanin², I. Pavkovic ${ }^{1, *}$, P. Kovacevic ${ }^{3}$, T. Kovacevic ${ }^{4}$ \\ University Hospital Clinical Center Banja Luka $-{ }^{1}$ Department of Nuclear Medicine and Thyroid Gland Disease \\ - Institute of Pathology - ${ }^{3}$ Department of Intensive Care Medicine - ${ }^{4}$ Department of Medical Supplies and \\ Pharmacy KC, Banja Luka, Bosnia and Herzegovina
}

\begin{abstract}
Background. Riedel thyroiditis is a rare, chronic inflammatory disease of the thyroid, characterized by a dense fibrosis that replaces normal thyroid parenchyma. In literature descriptions of individual cases of Riedel thyroiditis can be found.

Case report. We present an euthyroid patient with multinodular goiter who was diagnosed with Riedel thyroiditis using pathology. Imaging diagnostic methods (Ultrasonography, X-ray computed tomography, Magnetic resonance imaging, Radionuclide imaging) or various tests of thyroid function cannot confirm the diagnosis of Riedel thyroiditis.

Conclusion. Only level of IgG4 may be helpful for the diagnosis of Riedel thyroiditis, while pathology is used for its definitive confirmation.
\end{abstract}

Key words: Riedel thyroiditis, histopathology.

\section{INTRODUCTION}

Riedel thyroiditis, or Riedel's thyroiditis (RT), is a rare, chronic inflammatory disease of the thyroid gland characterized by a dense fibrosis that replaces normal thyroid parenchyma. The fibrotic process invades adjacent structures of the neck and extends beyond the thyroid capsule (1-4).

Description of individual cases of RT can be found in the literature. Only authors at the Mayo Clinic defined an incidence, which is $0.06 \%$ (1). When reviewing the histologic diagnosis of 56700 thyroidectomies performed at the Mayo Clinic (Rochester) between 1920 and 1984, Hay found only 37 cases of RT; the overall incidence in outpatients was 1.06/100000. Malotte et al. encountered only 1 case of RT in their review of more than 700 thyroid operations performed at Loma Linda University Medical Center (Loma Linda) during a 15year period (1).

\section{CASE REPORT}

In the Clinical Center Banja Luka (Republic of Srpska) in the period between 1994 - 2014, 4716 operations of the thyroid gland were performed and only one case of RT was found. We present here a rare case of RT in an young patient without an associated extracervical manifestation of multifocal fibrosclerosis.

A forty-three-year old woman has noticed "swelling" on the neck which felt quite solid. The patient had no other complaints. She did not use medications which can cause multifocal fibrosclerosis.

Thyroid function tests revealed thyroid stimulating hormone (TSH) level of $0.737 \mu \mathrm{IU} / \mathrm{mL}$ (reference range 0.27-4.20 $\mu \mathrm{IU} / \mathrm{mL}$ ); free T3 (FT3) of $2.0 \mathrm{pmol} / \mathrm{L}$ (reference range 1.30-3.10 nmol/L); free thyroxine (FT4) of $135 \mathrm{pmol} / \mathrm{L}$ (reference range 12-22 $\mathrm{pmol} / \mathrm{L}$ ). Serum calcitonin was $3.50 \mathrm{pg} / \mathrm{mL}$ (reference level less than $10 \mathrm{pg} / \mathrm{mL}$ ).

Fine-needle aspiration cytology provided material with scarce cellularity. In the material rare thyroid follicular cells with common cytological characteristics were seen. There was a small amount of colloids, fibroblasts, collagen fibers and inflammatory infiltrate. In the infiltrate lymphocytes, plasma cells and rare eosinophils were found (Fig. 1).

One month later the results of thyroid function test showed FT4 level of $22 \mathrm{pmol} / \mathrm{L}$; anti-thyroglobulin antibody (Tg ab) level of $43 \mathrm{IU} / \mathrm{mL}$ (reference range 0-115); thyroglobulin ( $\mathrm{Tg}$ ) level of $16.7 \mathrm{ng} / \mathrm{ml}$ (reference range 1.40-78.00); thyreoperoxidase antibodies (TPO ab) level of $10 \mathrm{IU} / \mathrm{mL}$ (to $70 \mathrm{U} / \mathrm{mL}$ normal, 70-130 U/ $\mathrm{mL}$ low positive); parathyroid hormone (PTH) level of $80 \mathrm{pg} / \mathrm{mL}$ (reference range 15-65 pg/mL). Biochemical testing showed total $\mathrm{Ca} 2.17 \mathrm{mmol} / \mathrm{L}$ (reference range 2.20-2.65 mmol/L); $\mathrm{Ca}^{++} 1.30 \mathrm{mmol} / \mathrm{L}$ (reference range $1.00-1.30 \mathrm{mmol} / \mathrm{L}$ ); phosphate $1.19 \mathrm{mmol} / \mathrm{L}$ (reference range $0.81-1.45 \mathrm{mmol} / \mathrm{L})$. The patient had elevated

*Correspondence to: Ivana Pavkovic MD, University Hospital Clinical Center Banja Luka, Department of Nuclear Medicine and Thyroid Gland Disease, Dvanaest beba bb Street, Banja Luka, Republic of Srpska, 78 000, Bosnia and Herzegovina, E-mail: ivanapavkovicbanjaluka@ gmail.com Acta Endocrinologica (Buc), vol. XII, no. 3, p. 339-343, 2016 

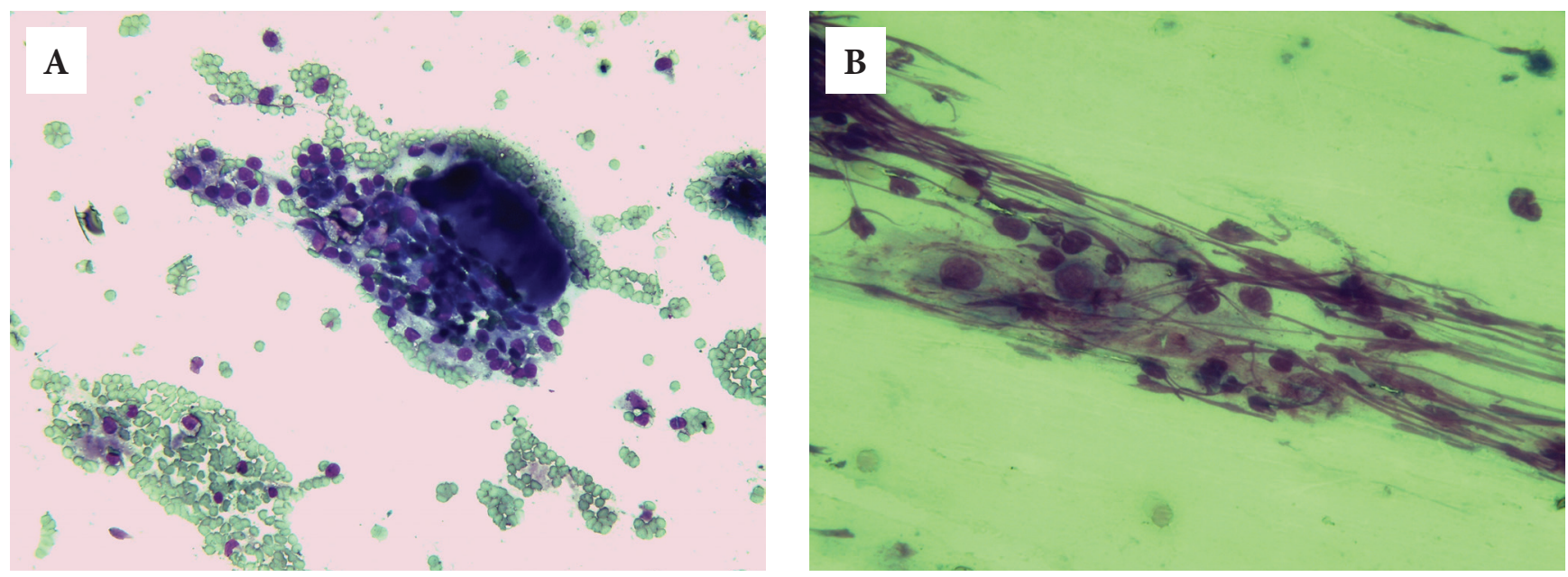

Figure 1. A and B. Thyroid follicular cells, colloid, fibroblasts, collagen fibers, lymphocytes and plasma cells (May-Grunwald Giemsa x 200).

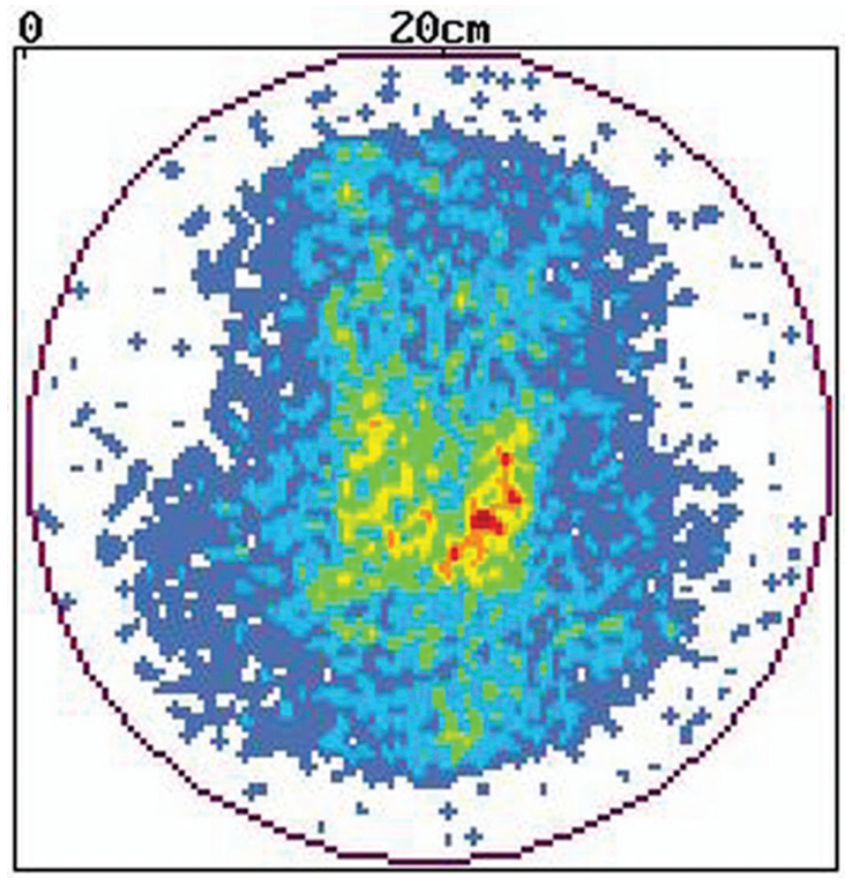

Figure 2. Thyroid scans with $74.20 \mathrm{MBq}{ }^{99 \mathrm{~m}} \mathrm{Tc}$.

levels of PTH and $\mathrm{Ca}^{++}$. However, subsequent testing showed normal values of PTH and $\mathrm{Ca}^{++}$. At that time in our institution we were not able to determine the level of IgG4.

\section{Imaging Studies}

\section{Ultrasonography}

Thyroid gland was normal size. Thyroid ultrasonography showed two hypoechogenic nodes in the right lobe of the thyroid gland with loss of clear demarcation from other tissue, and an inhomogeneous structure in the left lobe of the thyroid gland. The biggest node in the right lobe of the thyroid gland was $30 \mathrm{x} 17 \mathrm{~mm}$ in size. It was located at superior region of the right lobe of the thyroid gland. Second nodule was $11 \times 7 \mathrm{~mm}$ in size and it was located at inferior region of the right lobe of the thyroid gland.

\section{Nuclear scanning}

Nuclear thyroid scans with $74.20 \mathrm{MBq}$ ${ }^{99 \mathrm{~m}} \mathrm{Tc}$ demonstrated normal size of thyroid gland and inhomogeneous uptake at the right lobe. In the left lobe of the thyroid gland uptake was better than in the right lobe of the thyroid gland. Right lobe had irregular shape (Fig. 2).

The patient was referred to surgery. Four months later surgery was performed. PH diagnosis was RT.

\section{Pathological findings \\ Macroscopic findings}

The analyzed material is the thyroid gland. Dimensions of the left lobe of the thyroid gland are 30 $\mathrm{mm} \times 20 \mathrm{~mm} \times 10 \mathrm{~mm}$. The capsule is thickened, partly whitish and firm. Lobular architectonics of the thyroid gland is largely wiped out. In the slices of central parts of the thyroid gland, we can see unclear limited nodegray-white solid texture, $20 \mathrm{~mm} \times 15 \mathrm{~mm} \times 10 \mathrm{~mm}$ in size. A node is in continuity with whitish thickening of the capsule. Right lobe of the thyroid gland is $15 \mathrm{~mm}$ $\times 15 \mathrm{~mm} \times 10 \mathrm{~mm}$ in size. The capsule is occasionally whitish thickened and uneven. Parenchyma is largely wiped out. Slices show vaguely circumscribed node 15 $\mathrm{mm} \times 15 \mathrm{~mm} \times 10 \mathrm{~mm}$ in size. The node has the same characteristics as the left lobe.

\section{Histological picture}

Thyroid gland clips tissue was histologically examined and the same morphological changes are shown in both lobes. Parenchyma of the thyroid gland 

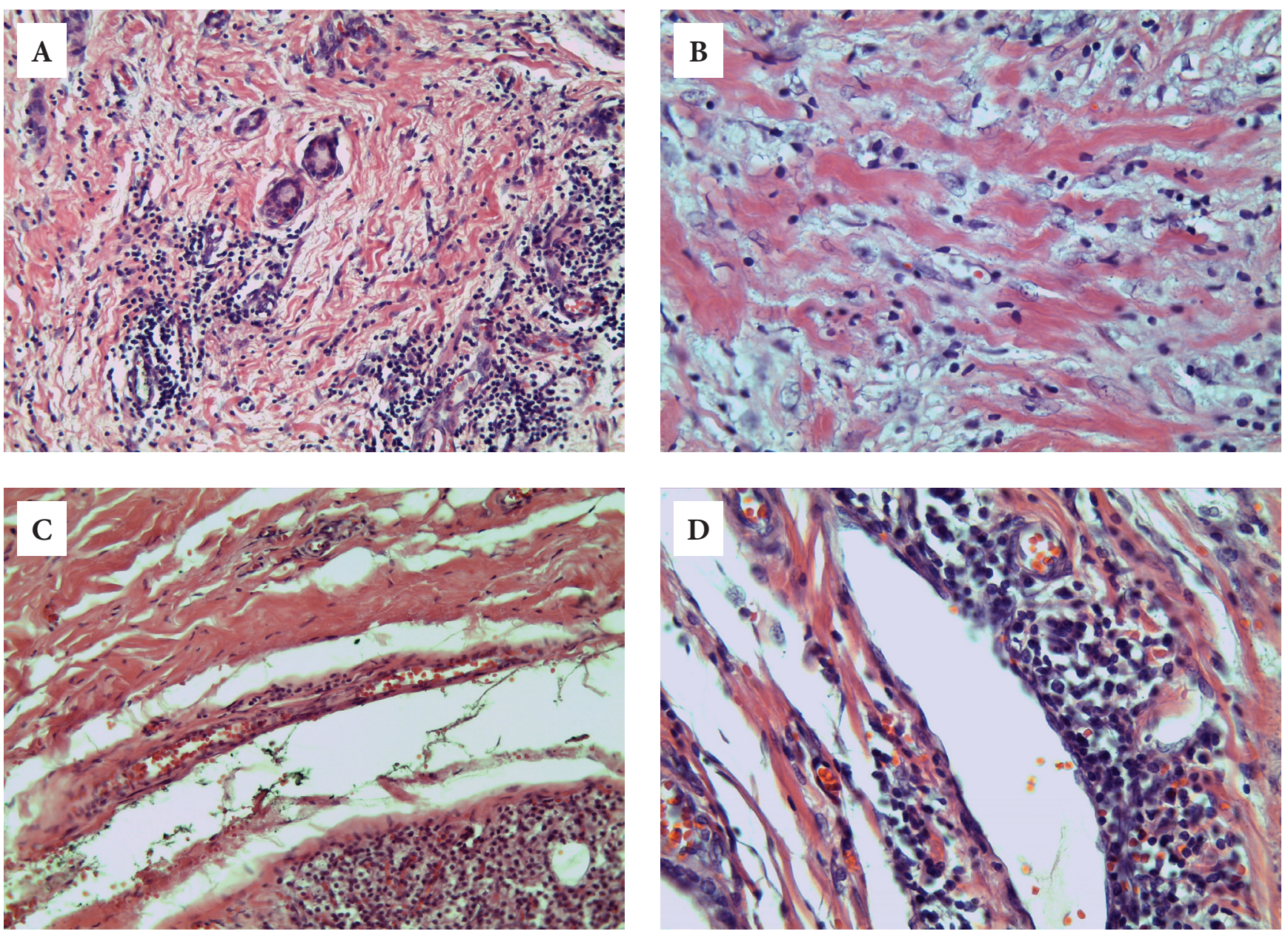

Figure 3. RT. A - Parenchyma of the thyroid gland was significantly reduced and replaced by connective tissue and inflammatory infiltrate. Between the cords of the connective tissue are rare follicles which are lined with a low, atrophic epithelium. Focal inflammatory infiltrate is built by lymphocytes, plasma cells, and rare eosinophilic granulocytes. B. - Connective tissue is constructed of fibroblasts and collagen fibers arranged anarchic tissue replaces the thyroid gland. C. - The strips of the connective tissue are located in the nearby tissue, outside thyroid. In the lower right corner of the image there is a part of the parathyroid gland embedded in connective tissue. D. - Inflammatory changes in veins. Inflammatory infiltrate is present, it is composed of lymphocytes and plasma cells and it is located around the veins and in the wall of veins Endothelial cells are enlarged and multiplied in some places, (haematoxylin and eosin x 200).

is largely reduced and replaced by connective tissue and inflammatory infiltrate (Fig. 3A). Multiplied connective tissue that extends from the capsule towards internal side of the thyroid gland can be seen in the clips. Connective tissue is constructed of fibroblasts, fibrocytes, and anarchic arranged collagen fibers (Fig. 3B). Connective tissue is located in the surrounding, perithyroid tissue (Fig. 3C). Between multiplied connective tissues are rare follicles, lined with low, atrophied follicular epithelium. Inflammatory infiltrate is thick, and it is built by lymphocytes, plasma cells, and rare eosinophilic granulocytes and neutrophils (Fig. 3D). The environment and the internal wall of small and medium-sized veins are built by the inflammatory infiltrate of lymphocytes plasma cells (Fig. 3D). Characteristic formation of lymphatic follicles and granulomas was not found in the examined materials.

The patient was twice hospitalized after surgical treatment in Clinical Center of Banja Luka. No fibrotic manifestations were found in other organs. There was no need for medical treatment with glucocorticoids and tamoxifen, since the patient was surgically managed with success.

\section{DISCUSSION}

The etiology of RT is unknown. A relatively new group of rare disorders, IgG4-related systemic disease (IgG4-RSD) includes RT. There are two theories of RT pathogenesis, first one is that RT results from an autoimmune process and the second is that RT might be a primary fibrotic disorder. However, 
IgG4-RSD may unify these two seemingly disparate etymologies $(3,4)$.

Morphologically, it is very important to make difference between RT and the fibrous variant of lymphocytes thyroiditis (Hashimoto) and other neoplastic lesion. The basic morphological characteristics that help in the differentiation of RT from fibrous variant of lymphocyte thyroiditis are: extrathyroid fibrosis, loss of lobular architectonics of the thyroid gland, the absence of lymphoid follicles and follicular oncocyte transformation of cells, the presence of vasculitis. Neoplastic processes that need to be differentiated are anaplastic carcinoma, sclerosing papillary carcinoma, Hodgkin's lymphoma (sclerosis nodularis), solitary fibrous tumors, different histological types of mesenchymal tumors. The differentiation is often necessary for using additional immunohistochemical methods (5).

On the first examination our patient was euthyroid. That was very important, because thyroid function can be normal, in hyperfunction and in hypofunction which depends on the extent to which the normal thyroid gland has been replaced by fibrotic tissue. Most patients are euthyroid, but hypothyroidism is noted in approximately $30 \%$ of cases. Rarely, hyperthyroidism can occur, but this is probably secondary to a coexisting condition $(1,4)$.

The initial laboratory assessment of patients with only thyroid disease always should include the measurement of serum thyrotropin levels. Most patients with RT have normal thyrotropin levels and one third have an elevated thyrotropin level. Also, it is very important to assess levels of $\mathrm{Tg} a b$ and TPO $\mathrm{Ab}$ which might be elevated, usually at a low titers, in a patient with RT (1). In our patient, levels of TPO $a b$, $\mathrm{Tg}$ ab and $\mathrm{Tg}$ were in reference range.

Some authors describe the connection between autoimmune process in parathyroid glands and hyperparathyroidism as well as the connection between autoimmune process in the thyroid gland and hyperparathyroidism (6). In our patient levels of PTH and $\mathrm{Ca}^{++}$were mildly elevated. We did not perform further examinations since control levels of PTH and $\mathrm{Ca}^{++}$in some subsequent measuring were in reference range, and pathological findings did not show any parathyroid changes.

Some experts say that RT is not primarily a thyroid disease. One theory is that RT is a manifestation of the multifocal fibrosclerosis systemic disorder. Approximately one third of RT cases are associated with clinical findings of multifocal fibrosclerosis at the time of diagnosis $(1,4)$. Our patient did not have manifestation of multifocal fibrosclerosis. Probably, this was primarily a thyroid disease, because we were not able to find extracervical manifestation of multifocal fibrosclerosis such as retroperitoneal fibrosis, mediastinal fibrosis, orbital pseudotumor, pulmonary fibrosis, sclerosing cholangitis, lacrimal gland fibrosis, fibrosing parotitis.

Giampaolo and colleagues described patients who developed multifocal fibrosclerosis after using certain medications. Patient's drug history might be related etiologically to RT. Medications that might be related to RT include methysergide, serotonin, lysergic acid, ergotamine, dihydroergotamine, hydralazine, methyldopa, acetazolamide, phenacetin and $\beta$ - blockers (1). Our patient did not use any of these medications.

Ultrasound cannot distinguish RT from other forms of thyroiditis. Hypoechogenicity is found in all inflammatory disorders of the thyroid gland (1). In our case findings were not specific, which corresponds to the data from the literature.

Radionuclide imaging of the thyroid gland with Tc-99 or 123/131-I is not usually indicated in euthyroid individuals with a palpable thyroid mass. The same images can be found in different forms of chronic thyroiditis (4). This confirms the previous argument, because nuclear thyroid scan in our patient showed inhomogeneous uptake at the right lobe.

Results of fine-needle aspiration are usually nondiagnostic. Material obtained by puncture is usually hypocellular; it contains rare follicular cells, fragments of connective tissue and scarce inflammatory infiltrate. In most cases it is interpreted as nondiagnostic (7). According to findings literature showed that $9.3 \%$ cases of fine needle aspiration cytology had disagreements. FNAC was from the thyroid, neck (soft tissue and lymph nodes), salivary gland and liver (8). In our case results of fine-needle aspiration did not refer to RT.

Definitive diagnosis is made only by histopathology, using open biopsy, or after decompressive goiter surgery performed for clinical symptoms (4).

MRI does not help in RT identification. On both T1- and T2- weighted protocols we can reveal hypointense images. Only after gadolinium administration we can observe a spectrum from little to marked homogeneous enhancement (5). Ali Ozgena and Aysenur Cilaa say that no other entity causes diffuse decreased enhancement after gadolinium administration on MRI imaging or administration of iodinated contrast medium on CT. This theory may be 
of importance for the diagnosis of RT (9).

Fludeoxyglucose F 18 positron emission tomography (FDG-PET) can trace effects of corticosteroid therapy in a patient with RT and multifocal fibrosclerosis (1). This diagnostic tool could be used for the diagnosis of diseases of the thyroid gland, but it is not suitable for routine examination.

Arowolo and others described a case report of patient with RT and non-resectable left lobe of thyroid gland. Patient was successfully managed with medical treatment using oral glucocorticoid, tamoxifen and L-thyroxine. There is no consensus on medical treatment. Therapy usually starts with glucocorticoids, mostly prednisolone. In case of treatment failure, it is necessary to use tamoxifen alone or in combination with glucocorticoids (11, 12). Tamoxifen induces transformation of growth factor beta, which inhibits fibroblast proliferation, so did not exhibit antiestrogen activity in RT. Hence oral treatment was not necessary in our patient, due to the fact that the patient was treated surgically with success.

This case was the only one reported out of 4716 operated patients monitored in a time period from 1994-2014, hence it is considered special. Also this was one of rare cases in which there was no systemic display of multifocal fibrosclerosis.

\section{Conflict of interest}

The authors declare that they have no conflict of interest concerning this article.

\section{References}

1. Papi G, Li Volsi VA. Current Concepts on Riedel Thyroiditis. Am J Clin Pathol 2004; 121:S50-S63.

2. Gyu Y P, Young S L, Soon W H. Hang-Seok Ch. Cheong S P. A case of Riedel's thyroiditis. J Korean Surg Soc 2012; 82(5):317320.

3. Pusztaszeri M, Triponez F, Pache JC, Bongiovanni M. Riedel's thyroiditis with increased IgG4 plasma cells: evidence for an underlying IgG4-related sclerosing disease. Thyroid 2012; 22(9):964-8.

4. James V, Hennessey. Riedel's Thyroiditis: A Clinical Review. J Clin Endocrinol Metab 2011; 96(10):3031-3041.

5. Biddinger PW. Thyroiditis. In: Nikiforov YE, Biddinger PW, Thompson LDR. Diagnostic Pathology and Molecular Genetics of the Thyroid. First edition. Philadelphia: Lippincott Williams \& Wilkins 2009:40-60.

6. M. Therese Cating-Cabral, A. Claro Cabungcal, C. Vincent Villafuerte III, J. Añel-Quimpo. Primary hyperparathyroidism due to an intrathyroidal parathyroid adenoma associated with chronic lymphocytic thyroiditis. BMJ Case Reports 2012; doi:10.1136/bcr2012-006198.

7. Galera-Davidson H, Gonzales-Campora R. Thyroid. In: Bibbo M, Wilbur DC. Comprehensive Cytopathology. Fourth Edition. London: Elsevier Sanuders, 2015: 511- 544

8. Howlett DC, Harper B, Quante M, Berresford A, Morley M, Grant J, Ramesar K, Barnes S. Diagnostic adequacy and accuracy of fine needle aspiration cytology in neck lump assessment: results from a regional cancer network over a one year period. J Laryngol Otol. 2007; 121(6):571-579.

9. F Kosari, H M Tabriz, S Saniee. Diagnostic Fine Needle Aspiration in a Case of Riedel's thyroiditis. Iran J. Pathol 2009; (4):186-189.

10. P Kotilainen, L Airas, T Kojo, T Kurki, K Kataja, H Minn, $P$ Nuutila. Riedel's thyroiditis in a patient with multiple sclerosis. Neuroendocrinol Lett 2005; 26(1):67-68.

11. A Özgena, A Cilaa. Riedel's Thyroiditis in Multifocal Fibrosclerosis: CT and MR Imaging Findings. Am J Neuroradiol 2000; 21:320-321.

12. Arowolo OA, Ige FS, Odujoko O, Agbakwuru EA. Riedel's thyroiditis in a black African: A case report and review of literature. Niger J Clin Pract 2016;19:549-555. 